\title{
Embolization Tactics of Spinal Epidural Arteriovenous Fistulas
}

\author{
Abdulrahman Hamad Al-Abdulwahhab, $\mathrm{MD}^{1,2 *}$, Yunsun Song, $\mathrm{MD}, \mathrm{PhD}^{1 *}$, Boseong Kwon, $\mathrm{MD}^{1}$, \\ Dae Chul Suh, MD, PhD ${ }^{1}$ \\ ${ }^{1}$ Neurointervention Clinic, Department of Radiology, Asan Medical Center, University of Ulsan College of Medicine, Seoul, Korea \\ ${ }^{2}$ Diagnostic and Interventional Radiology Department, Imam Abdulrahman Bin Faisal University, King Fahd Hospital of the University, \\ Al-Khobar City, Saudi Arabia
}

Purpose: Spinal epidural arteriovenous fistulas (SEDAVFs) show an epidural venous sac often with venous congestive myelopathy (VCM) due to intradural reflux at a remote level to which a transarterial approach would be difficult. We present 12 cases of SEDAVF with VCM and describe 3 main tactics for effective transarterial embolization.

Materials and Methods: Among 152 patients with spinal vascular malformations diagnosed in our tertiary hospital between 1993 and 2019, 12 SEDAVF patients with VCM were included. Three different transarterial embolization tactics were applied according to the vascular configuration and microcatheter accessibility. We evaluated treatment results and clinical outcomes before and after treatment.

Results: Transarterial embolization with glue (20-30\%) was performed in all patients. The embolization tactics applied in 12 patients were preferential flow $(n=2)$, plug-and-push $(n=6)$, and filling of the venous sac $(n=4)$. Total occlusion of the SEDAVF, including intradural reflux, was achieved in 11 (91.7\%) of 12 patients, and partial occlusion was achieved in 1 patient. No periprocedural complications were reported. Spinal cord edema was improved in all patients for an average of 18 months after treatment. Clinical functional outcome in terms of the pain, sensory, motor, and sphincter scale and modified Rankin scores improved during a mean 25-month follow-up (6.3 vs. 3.3, $\mathrm{P}=0.002 ; 3.6$ vs. 2.3, $\mathrm{P}=0.002$, respectively).

Conclusion: Endovascular treatment for 12 SEDAVF patients with VCM achieved a total occlusion rate of $91.7 \%$ without any periprocedural complication. The combined embolization tactics can block intradural reflux causing VCM, resulting in overall good clinical outcomes.

Key Words: Arteriovenous fistula; Spinal cord vascular diseases; Embolization, therapeutic; Endovascular procedures

\section{INTRODUCTION}

Spinal epidural arteriovenous fistula (SEDAVF) is a rare type of vascular malformation increasingly identified by high-resolution 3-dimensional rotational angiography (3DRA), ${ }^{1,2}$ In the classification system of spinal arteriovenous shunt diseases, the concept of SEDAVF has emerged relatively recently, and has been reported as ventral epidural shunts, ${ }^{3}$ extradural arteriovenous fistulas (AVFs), ${ }^{4-6}$ or ventral epidural $\mathrm{AVFs}^{7}$; and finally, it is now commonly referred to as an epidural AVF. ${ }^{8}$

SEDAVFs are characterized by an epidural venous sac, usually located in the ventral epidural space, multiple

\section{Correspondence to: Dae Chul Suh, MD, PhD \\ Neurointervention Clinic, Department of Radiology, Asan Medical Center, University of Ulsan College of Medicine, 88, Olympic-ro 43-gil, Songpa-gu, Seoul 05505, Korea Tel: +82-2-3010-4366 \\ Fax: +82-2-3010-0090 \\ E-mail:dcsuh@amc.seoul.kr}

*Abdulrahman Hamad Al-Abdulwahhab and Yunsun Song contributed equally to the first authors.

Received: May 11, 2021

Revised: July 2, 2021

Accepted: July 28, 2021

\begin{abstract}
Copyright $\odot 2021$ Korean Society of Interventional Neuroradiology

This is an Open Access article distributed under the terms of the Creative Commons Attribution Non-Commercial License (http://creativecommons.org/licenses/by-nc/4.0) which permits unrestricted non-commercial use, distribution, and reproduction in any medium, provided the original work is properly cited.
\end{abstract}

pISSN 2093-9043 eISSN 2233-6273 
bilateral feeders, and the absence of horizontal T-sign, which is a typical sign of spinal dural arteriovenous fistula (SDAVF). ${ }^{9}$ SEDAVFs result from direct venous arterialization of the epidural venous plexus by the epidural arterial branches of the ascending cervical, vertebral, intercostal, lumbar, or sacroiliac arteries. ${ }^{9}$ The shunt flow drains first into the epidural venous sac and then to the paravertebral vein and, in some cases, retrogradely into the intradural vein. ${ }^{5}$ In contrast, SDAVF is fed by a feeder in most cases and located dorsally within the dural sleeve between the radiculomeningeal artery and radicular or bridging veins without forming a dilated epidural venous sac. $^{10-12}$

The main goal of treatment is to alleviate neurological symptoms caused by venous congestive myelopathy (VCM) by occluding the epidural venous pouch or the culprit vein that causes intradural reflux. Endovascular treatment with a transarterial approach can be performed if the arterial feeders supplying a small epidural venous sac have a relatively straight course, especially in the non-osseous type of SEDAVF. However, SEDAVF with multiple feeders and a venous pouch may require different strategies from the conventional techniques using free flow or an induced wedge method, which are techniques for SDAVF.11,13,14 Transvenous embolization may be selectively performed in cases involving difficult arterial access, large epidural venous sacs, or azygous drainage. Nevertheless, it is often technically challenging depending on the venous outflow restriction and variable paths to the paraspinal longitudinal vein. A ventrally-located orientation of SEDAVF with a large confluent venous pouch, which embryologically mimics a cavernous sinus DAVF, may preclude a surgical approach. ${ }^{3,15,16}$

We present 12 cases of SEDAVF with VCM and describe 3 different embolization tactics to overcome the distance from the microcatheter tip to the epidural venous sac or intradural reflux point. We also evaluated clinical outcomes based on the pain, sensory, motor, and sphincter (PSMS) scale ${ }^{17}$ and modified Rankin score (mRS) before and after endovascular treatment.

\section{MATERIALS AND METHODS}

\section{Patient Selection}

The Institutional Review Board at our medical center approved this retrospective study and waived the requirement to obtain written informed consent from the patients.
Among 152 spinal vascular malformations from a prospectively maintained database from a single tertiary hospital between January 1993 and September 2019, 21 patients were diagnosed and confirmed for a SEDAVF by digital subtraction spinal angiography. We included 12 non-osseous SEDAVF patients with VCM, excluding 4 without VCM and 5 osseous SEDAVF, in which transvenous coiling was the main procedure of choice for the large venous sac in the bony defect. The 12 patients included 9 males and 3 females with a mean age of 59 years (range, 33-83 years). Some of these patients have been previously described. Patient data, including clinical presentation, neurologic assessment, and follow-up results, were obtained from the database connected to the electronic medical record system.

We classified the patient presentations as myelopathy, radiculopathy, or myeloradiculopathy. ${ }^{2}$ Myelopathy was defined as a neurologic deficit related to spinal cord disease and included motor and sensory deficits, gait disturbance, or sphincter dysfunction. Radiculopathy was defined as a range of symptoms produced by pinching a nerve root in the spinal column following specific dermatomal distribution. Finally, radiculomyelopathy was defined as a range of symptoms, including those of radiculopathy and myelopathy. We categorized functional disability according to the PSMS scale ${ }^{17}$ and mRS. The scores were based on the records of neurologic examinations of neurologists or neurointerventionists.

\section{Imaging Diagnosis}

SEDAVF was defined as an arteriovenous shunt in the spinal epidural space fed by epidural arterial branches, draining primarily into the epidural venous sac and subsequently into the paravertebral veins with or without intradural reflux. Digital subtraction spinal angiography (Artis zee; Siemens, Forchheim, Germany) with selective angiography or 3DRA was used for localization and characterization of the SEDAVF, including the relationship between feeding arteries, epidural venous sacs, epidural venous drainage, and the point of intradural reflux and the venous drainage pattern. 2,18,19

Spinal magnetic resonance imaging (MRI) was performed for the initial assessment of the disease. Axial and sagittal images of T1- and T2-weighted sequences with or without contrast enhancement were obtained with $1.5 \mathrm{~T}$ or $3 \mathrm{~T}$ systems. VCM was defined when MRI revealed spinal cord edema and perimedullary flow voids. Compressive myelopathy was defined as when there was a cord signal change due to cord compression from an epidural lesion. Patients who needed 
imaging evaluation clinically after treatment underwent follow-up MRI. ${ }^{20}$ Follow-up spinal MRI was performed to assess disease outcomes.

\section{Endovascular Treatments with 3 Embolization Tactics}

Endovascular embolization via the transarterial approach was considered primarily when the vascular approach to the fistular site was accessible. The location of the intradural reflux was the main target for embolization to prevent retrograde venous drainage leading to VCM. We approached the fistula point as close as possible using a low-profile microcatheter to achieve a wedged position, and no other flow control techniques were used. We used glue that was a mixture of N-butyl-2-cyanoacrylate (Histoacryl; B. Braun, Melsungen, Germany) mixed with Lipiodol (Guerbet, Roissy, France). We also used detachable or pushable coils to reduce collateral inflow or support the microcatheter in a sharp curve.

Three technical strategies, including preferential flow, plugand-push, and filling of the venous sac, were considered depending on the distance from the microcatheter tip to the epidural venous sac or intradural reflux point. The prefer-
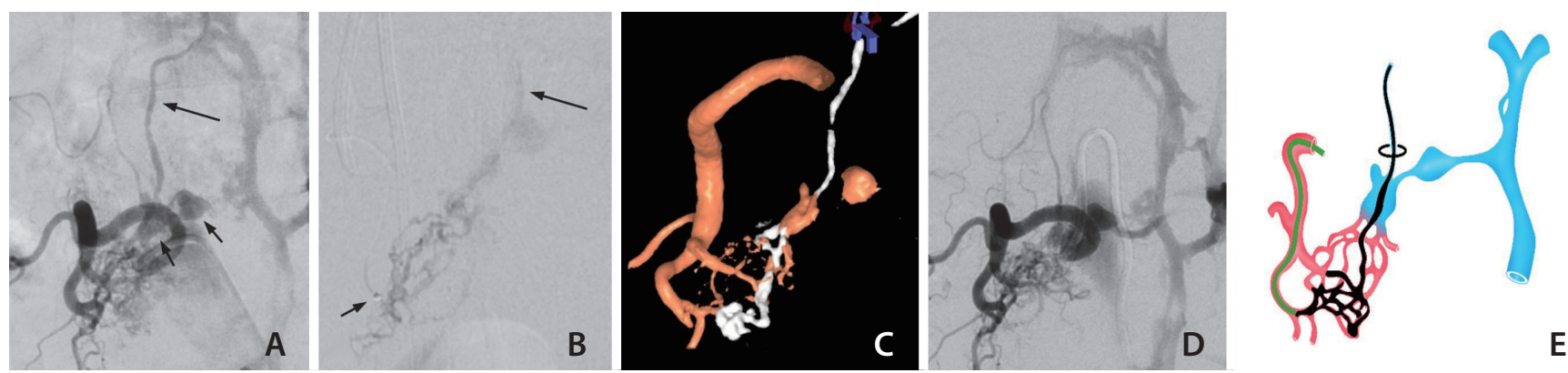

Fig. 1. Prefrential flow technique for a ventrally located spinal epidural arteriovenous fistula at the L2 level (patient 10). Anteroposterior view of the right second lumbar segmental arteriography (A) shows multiple feeders and a dilated venous sacs (short arrows) and intradural venous reflux (long arrow) causing congestive venous myelopathy. (B) Selective angiography was performed via a microcatheter (short arrow) to identify preferential flow into the intradural venous reflux (long arrow). The same view, (C) obtained after glue embolization. Note the glue cast (white color in $\mathbf{C}$ ) obliterating the intradural venous reflux by the preferential flow. Post-embolization angiography (D) shows no filling of the intradural venous reflex despite the remaining epidural shunts. (E) The schematic diagram shows the preferential flow technique requires an adjusted selection of a proper microcatheter (green color) site to deliver embolic material into the intradural venous reflux. Black color: glue cast, Black ring: intradural venous reflux point, Green color: microcatheter.
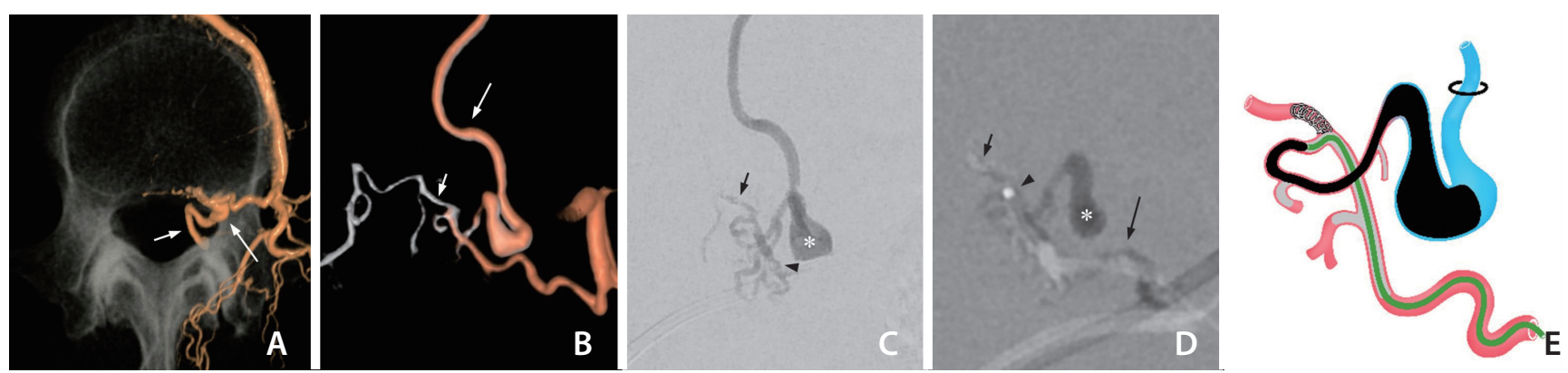

Fig. 2. Plug-and-push technique for spinal epidural arteriovenous fistula at the $L 3$ level (case 12). (A) Volume-rendering 3-dimensional (3D) spinal angiography showing the dilated venous sac (long arrow) located in the left ventrolateral epidural space and the connected intradural vein (short arrow). (B) Fusion 3D images of both sides showing ventral epidural collateral via the contralateral dorsal somatic artery (short arrow) and intradural reflux point (long arrow). (C) Selective angiography at the proximal feeder (arrowhead) showing multiple fine arteries that were not visible in the 3D angiography converged to the venous sac (asterisk) and a retrograde filling of the contralateral feeder (arrow), which was protected by a coil. (D) After coil protection of the epidural collateral (short arrow), the glue was injected at the position (arrowhead) as distal as possible to penetrate the dilated venous sac (asterisk) using the plug-and-push technique. Note the proximal plug at the proximal segmental artery (long arrow). (E) The schematic diagram shows the plug-and-push technique in front of the shunt to make proximal and side-branch plugs (grey color) so that the glue is then advanced against rather high pressure from the other feeders to fill the epidural venous sac. Black color: glue cast, Black bordered mesh: coil to prevent collateral inflow, Black ring: intradural venous reflux point, Green color: microcatheter, Grey color: plugs against the proximal feeder pedicle as well as the other feeders to propagate glue cast into the epidural venous sac. 
ential flow technique is used when the arterial feeder comprises several fine channels, and a further approach is not possible (Fig. 1). On a selective angiography or fluoroscopic exploration, the position of the microcatheter tip should be adjusted to reach the best point where the flow preferentially enters the intradural vein. Then, the embolic agent is released continuously from the tip of the microcatheter under the antegrade preferential free flow until the opening of the intradural venous drainage is occluded. The plug-and-push technique is used when there are inflows from other feeders between the microcatheter tip and the fistula even though the microcatheter advanced as close as possible to the fistula (Fig. 2). To prevent the flow of a relatively high-pressure gradient from other feeders and to form a plug, several discontinuous injections of a small volume of embolic material are required until reaching the opening of the intradural vein. A venous sac filling technique is used where there is no inflow from another feeder in the path (Fig. 3). Embolic material is continuously injected into the large confluent epidural venous sac to obliterate shunt flow and deliver the material to the opening of the intradural venous drainage to prevent possible recruitments of venous inflow from other feeders mainly located in the large epidural venous (fistular) sac.

Complete occlusion was defined as either occlusion of the opening of the culprit vein where intradural reflux occurred or complete obliteration of the epidural venous (fistular) sac from which connected to remote intradural reflux point, and to which every feeder was recruited. Partial occlusion was defined as residual flow into the intradural vein or delayed opacification of the fistula in the venous phase.

\section{Outcome Assessments and Statistical Considerations}

We used the PSMS scale and mRS to assess the patients' neurologic symptoms and functional abilities at every follow-up. ${ }^{17}$ We evaluated the clinical outcome by comparing the scores assessed immediately before the procedure and the most recent outpatient follow-up. The matched ordinal scores of mRS and PSMS before and after the procedure were compared using the Wilcoxon signed-rank test. A P-value $<0.05$ was considered to be statistically significant. Statistical analyses were performed using STATA version 15.1 (StataCorp LP, College Station, TX, USA).

\section{RESULTS}

\section{Baseline Characteristics}

The mean duration from symptom onset to diagnosis was 10 months (range, 1 to 36 months) (Table 1). All patients had intradural venous drainage and complained of lower
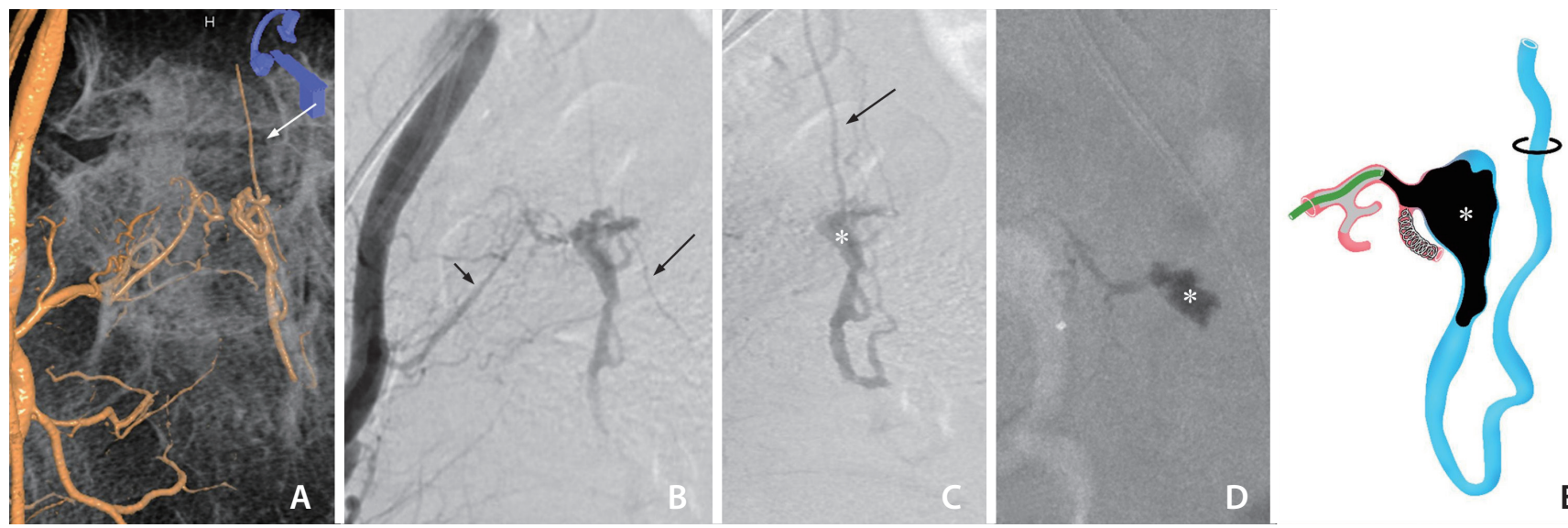

Fig. 3. Filling of venous sac technique for spinal epidural arteriovenous fistula at the S1 level (case 1). (A) Angiogram of the right internal iliac artery showing an epidural shunt at the left S1 level draining into a dilated venous sac regurgitating into the intradural venous reflux (arrow). (B) Selective right lateral sacral arteriography showing a feeder (short arrow). Note filling of the opposite dorsal somatic branch via the epidural collateral (long arrow). (C) Selective microcatheter angiography at the wedged position after coil protection for the epidural channel shows a venous sac (asterisk) connected to the radicular vein with intradural venous reflux (arrow). (D) Glue cast filling the venous sac (asterisk) obliterated the fistula using a continuous venous sac filling strategy. (E) The schematic diagram of filling the dilated epidural venous sac with rather low resistance, which drains into the culprit vein. The intradural reflux point is at a different segmental level (black ring). Black color: glue cast, Black bordered mesh: coil to prevent collateral inflow, Black ring: intradural venous reflux point, Green color: microcatheter, Grey color: plugs against the proximal feeder pedicle as well as the other feeders to propagate glue cast into the epidural venous sac (asterisk). 


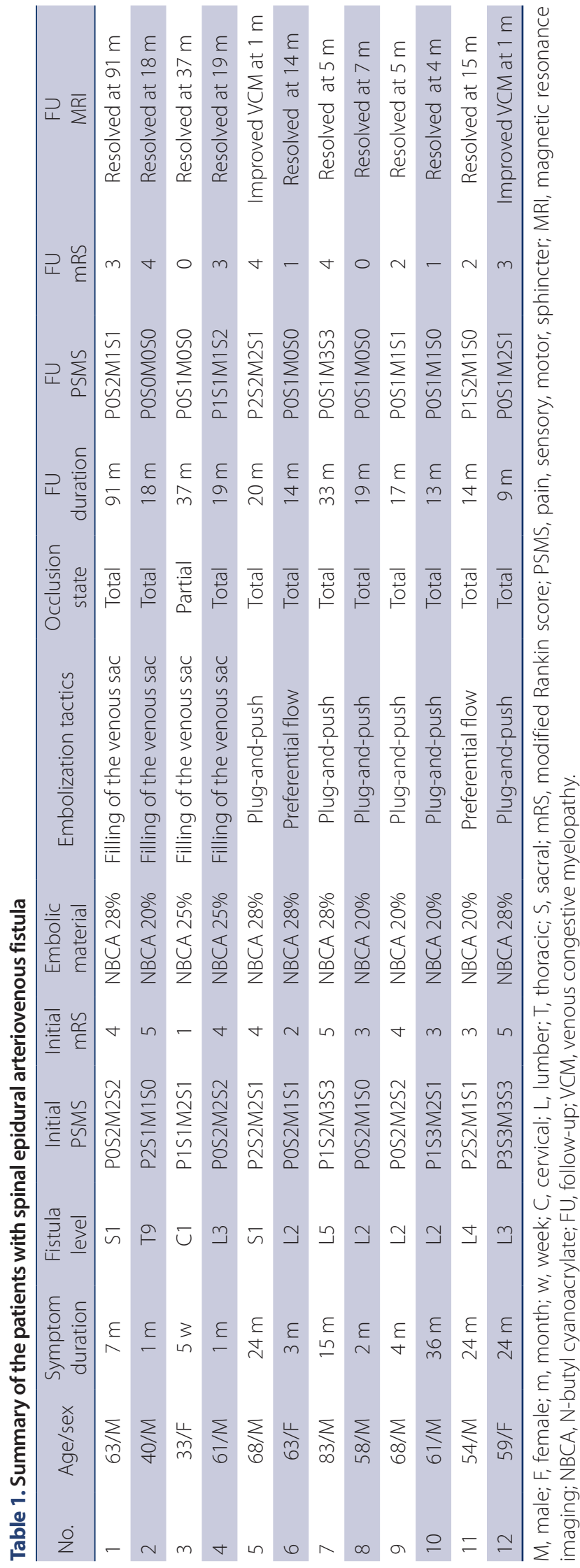

extremity weakness or sphincter dysfunction due to congestive myelopathy. One patient (case 5) had a history of trauma ( $L 1$ vertebral body fracture), and 2 had a history of L4-5 discectomy (case 10 and 11).

\section{Imaging Diagnosis and Endovascular Treatment}

The diagnosis was confirmed by selective angiography or 3DRA. In all patients, an epidural venous sac with both intradural and extradural drainages was observed. The fistula levels were distributed in the cervical $(n=1)$, thoracic $(n=1)$, lumbar $(n=8)$, and sacral $(n=2)$ regions. The locations of the venous sac in the epidural space were ventral in 7 patients and lateral in 5 patients. All patients had spinal cord edema on spinal MRl, suggesting VCM.

In all patients, transarterial embolization with 20-30\% glue was performed. Adjuvant coils were used to prevent collateral inflow from opposite or different-level segmental arteries $(n=3)$ and support the microcatheter at the sharp turning point of the branch $(n=1)$. Three embolization techniques were employed by using preferential flow $(n=2)$, plug-andpush $(n=6)$, and filling of the venous sac $(n=4)$ (Figs. 1-3, respectively).

Complete occlusion of the fistula or intradural drainage vein was achieved in 11 patients (91.7\%). In 1 patient with partial occlusion (case 3), delayed opacification of the intradural vein and much decreased shunt flow were noticed after embolization; however, minimal residual flow remained due to insufficient glue penetrance. No periprocedural complications were reported.

\section{Outcomes}

The mean clinical follow-up duration after treatment was 25 months (range, 9-91 months). The PSMS and mRS scores improved in all patients except for 1 patient (case 5) who did not show improvement despite complete occlusion of the fistula. The overall mean PSMS and mRS scores were significantly decreased at follow-up (6.3 vs. 3.3, $P=0.002 ; 3.6$ vs. 2.3, $\mathrm{P}=0.002$, respectively). Regarding MRI findings, spinal cord edema completely disappeared $(n=10)$ or improved $(n=2)$ at a mean of 18 months follow-up after the embolization.

\section{DISCUSSION}

Endovascular treatment can be an effective method for the treatment of a SEDAVF. In previous reports, the complete 
obliteration rates of a SEDAVF ranged from $73.3 \%$ to $94.4 \%$, and neurological symptoms improved in up to $91 \%$ of cases after endovascular treatment. ${ }^{21,22}$ Our study of 12 SEDAVF patients with VCM showed comparable results with a complete obliteration rate of $91.7 \%$, and clinical outcomes in terms of PSMS and mRS scores were improved in $91.7 \%$ of the patients. In a patient whose symptoms did not improve despite good treatment results, the time from symptom onset to treatment was 24 months, possibly resulting in irreversible spinal cord damage. Considering our result, conceptualization of the 3 embolization techniques could help in getting better outcomes. Although a recently published study of SEDAVF reported that the initial treatment success rate of endovascular treatment (69\%) was lower than that of surgery (92.5\%), ${ }^{23}$ endovascular treatment using these embolization tactics may still be a good option considering its minimal invasiveness and comparable clinical outcomes. ${ }^{21,22,24}$

We applied the 3 tactics (preferential flow, plug-and-push, and filling of the venous sac) depending on the microcatheter position and the relationship with other feeders. We performed 3DRA and obtained fusion images from the opposite or adjacent levels to understand the relationships among the feeders reaching the fistulas and venous drainage, especially into the intradural components. The distance from the epidural venous sac to the point of intradural reflux is usually far remote beyond the fistular sac and is crucial for the treatment during the transarterial approach. The culprit vein that shows intradural venous reflux causing VCM should be blocked even in situations where the lesion is too extensive or difficult to occlude all the fistular flows. When the epidural shunt remains, however, it may recruit another intradural venous drainage, especially when the epidural vein receiving the feeder extends over several vertebral levels. When there are multiple feeders, the feeders usually converge to a large epidural venous fistular sac for the embolization target, except in some cases in which numerous feeders diverge into the venous channels without forming a single dilated epidural venous fistular sac. Therefore, embolization may require different strategies in each case using different embolization tactics.

We essentially tried to achieve a wedged position of the microcatheter, and in doing so arrest antegrade flow of the feeder by blocking the vessel lumen with a microcatheter tip. However, flow control techniques such as an induced-wedge or proximal coil-protected technique using another microcatheter and coil may be a good option when the ideal microcatheter position cannot be achieved. ${ }^{11,25}$ The plug-and-push technique that we used is mainly used to block the inflow from other feeders, unlike the technique using onyx, which generally prevents reflux into the parent artery. A combined technique or transvenous embolization may be necessary in cases involving large epidural venous sacs fed by numerous feeders with epidural or paravertebral drainage or in an osseous-type SEDAVF. ${ }^{26}$

Embolic materials seem to be varied according to the operator's preference. We preferred to use a 20-30\% concentration glue for easy control, short injection time, better visibility, and more thrombogenicity than others. In contrast, other centers preferred onyx embolization material over glue during trans-arterial embolization, which tends to permeate the venous side of the fistula and fill other arterial feeding vessels in a retrograde fashion. Also, Onyx can be delivered more slowly than glue with a more controlled injection under fluoroscopy. The superior penetration of the fistula with Onyx, when compared with glue, allows one to avoid distal catheterization. Onyx is a non-thrombogenic embolic material that allows for better packing of the epidural venous sac without the risk of thrombus formation. ${ }^{27}$

One limitation of this study was its retrospective design with a relatively small number of patients due to the rarity of vascular malformation and shared data with previous publications. Furthermore, not all patients underwent postoperative follow-up by spinal angiography, as we did not perform routine imaging unless clinical improvement was not shown.

\section{CONCLUSION}

Endovascular treatment for 12 SEDAVF patients with VCM achieved a total occlusion rate of $91.7 \%$ without any periprocedural complication. The 3 different embolization tactics (preferential flow, plug-and-push, and filling of the venous sac) were used to effectively block intradural reflux causing VCM. Clinical outcomes were improved in all patients except for 1 patient who did not show improvement despite good treatment results, possibly due to a late presentation after symptom onset.

\section{Fund}

This work was supported by the National Research Foundation of Korea (NRF) grant funded by the Korean government (MSIT) (No. 2018R1A2B6003143). 


\section{Ethics Statement}

The Institutional Review Board at our medical center approved this retrospective study and waived the requirement to obtain written informed consent from the patients.

\section{Conflicts of Interest}

DCS has been the Editor-in-Chief of the Neurointervention since 2018. No potential conflict of interest relevant to this article was reported.

YS has been the Assistant Editor of the Neurointervention since 2019. No potential conflict of interest relevant to this article was reported.

No other authors have any conflict of interest to disclose.

\section{Author Contribution}

Concept and design: DCS. Analysis and interpretation: AHA, YS, BK, and DCS. Data collection: AHA, YS, and BK. Writing the article: AHA, YS, and DCS. Critical revision of the article: YS and DCS. Final approval of the article: AHA, YS, BK, and DCS. Obtained funding: DCS. Overall responsibility: DCS.

\section{ORCID}

Abdulrahman Hamad Al-Abdulwahhab:

https://orcid.org/0000-0003-4398-7864

Yunsun Song: https://orcid.org/0000-0003-4738-0533

Boseong Kwon: https://orcid.org/0000-0002-6113-9730

Dae Chul Suh: https://orcid.org/0000-0003-1561-5596

\section{REFERENCES}

1. Byun JS, Tsang ACO, Hilditch CA, Nicholson P, Fang YB, Krings T, et al. Presentation and outcomes of patients with thoracic and lumbosacral spinal epidural arteriovenous fistulas: a systematic review and meta-analysis. J Neurointerv Surg 2019;11:95-98

2. Park JE, Koo HW, Liu H, Jung SC, Park D, Suh DC. Clinical characteristics and treatment outcomes of spinal arteriovenous malformations. Clin Neuroradiol 2018;28:39-46

3. Geibprasert S, Pereira V, Krings T, Jiarakongmun P, Toulgoat F, Pongpech S, et al. Dural arteriovenous shunts: a new classification of craniospinal epidural venous anatomical bases and clinical correlations. Stroke 2008;39:2783-2794

4. Rangel-Castilla L, Holman PJ, Krishna C, Trask TW, Klucznik RP, Diaz OM. Spinal extradural arteriovenous fistulas: a clinical and radiological description of different types and their novel treatment with Onyx. J Neurosurg Spine 2011;15:541-549
5. Takai K, Taniguchi M. Comparative analysis of spinal extradural arteriovenous fistulas with or without intradural venous drainage: a systematic literature review. Neurosurg Focus 2012;32:E8

6. Takai K. Spinal arteriovenous shunts: angioarchitecture and historical changes in classification. Neurol Med Chir (Tokyo) 2017;57:356-365

7. Kiyosue H, Tanoue S, Okahara M, Hori Y, Kashiwagi J, Mori H. Spinal ventral epidural arteriovenous fistulas of the lumbar spine: angioarchitecture and endovascular treatment. Neuroradiology 2013;55:327-336

8. Lenck S, Nicholson P, Tymianski R, Hilditch C, Nouet A, Patel $K$, et al. Spinal and paraspinal arteriovenous lesions. Stroke 2019;50:2259-2269

9. Kiyosue H, Matsumaru Y, Niimi Y, Takai K, Ishiguro T, Hiramatsu M, et al.; JSNET Spinal AV Shunts Study Group. Angiographic and clinical characteristics of thoracolumbar spinal epidural and dural arteriovenous fistulas. Stroke 2017;48:3215-3222

10. Cho SH, Suh DC. Transdural segment of the radicular vein in spinal dural arteriovenous fistula. Neurointervention 2017;12:5758

11. Suh DC, Cho SH, Park JE, Liu H, Jung SC. Induced-wedge technique to improve liquid embolic agent penetration into spinal dural arteriovenous fistula. World Neurosurg 2016;96:309-315

12. Gemmete JJ, Chaudhary N, Elias AE, Toma AK, Pandey AS, Parker RA, et al. Spinal dural arteriovenous fistulas: clinical experience with endovascular treatment as a primary therapy at 2 academic referral centers. AJNR Am J Neuroradiol 2013;34:19741979

13. Su IC, terBrugge KG, Willinsky RA, Krings T. Factors determining the success of endovascular treatments among patients with spinal dural arteriovenous fistulas. Neuroradiology 2013;55:13891395

14. Zhao LB, Shim JH, Lee DG, Suh DC. Two microcatheter technique for embolization of arteriovenous fistula with liquid embolic agent. Neurointervention 2014;9:32-38

15. Song Y, Cho SH, Lee DW, Sheen JJ, Shin JH, Suh DC. Osseous versus nonosseous spinal epidural arteriovenous fistulas: experiences of 13 patients. AJNR Am J Neuroradiol 2019;40:129-134

16. Suh DC. Where did the dura mater come from? Neurointervention 2020;15:2-3

17. Suh DC, Song Y, Park D, Han M, Lim YM, Park JE, et al. New grading system for the clinical evaluation of patients with spinal vascular lesions. Neuroradiology 2018;60:1035-1041

18. Jung SC, Song Y, Cho SH, Kim J, Noh SY, Lee SH, et al. Endovascular management of aneurysms associated with spinal arteriovenous malformations. J Neurointerv Surg 2018;10:198-203 
19. Adrianto Y, Yang KH, Koo HW, Park W, Jung SC, Park JE, et al. Concomitant origin of the anterior or posterior spinal artery with the feeder of a spinal dural arteriovenous fistula (SDAVF). J Neurointerv Surg 2017;9:405-410

20. Lee CS, Pyun HW, Chae EY, Kim KK, Rhim SC, Suh DC. Reversible aggravation of neurological deficits after steroid medication in patients with venous congestive myelopathy caused by spinal arteriovenous malformation. Interv Neuroradio/ 2009;15:325-329

21. Nasr DM, Brinjikji W, Clarke MJ, Lanzino G. Clinical presentation and treatment outcomes of spinal epidural arteriovenous fistulas. J Neurosurg Spine 2017;26:613-620

22. Huang W, Gross BA, Du R. Spinal extradural arteriovenous fistulas: clinical article. J Neurosurg Spine 2013;19:582-590

23. Takai K, Endo T, Yasuhara T, Seki T, Watanabe K, Tanaka Y, et al. Microsurgical versus endovascular treatment of spinal epidural arteriovenous fistulas with intradural venous drainage: a multicenter study of 81 patients. J Neurosurg Spine 2020;33:381-391

24. Brinjikji W, Cloft H, Lanzino G. E-029 clinical, angioarchitectural and treatment characteristics of spinal dural arteriovenous fistulas versus spinal epidural arteriovenous fistulas. J Neurointerv Surg 2020;12:A42

25. Park KY, Kim JW, Kim BM, Kim DJ, Chung J, Jang CK, et al. Coil-protected technique for liquid embolization in neurovascular malformations. Korean J Radio/ 2019;20:1285-1292

26. Suh DC, Choi CG, Sung KB, Kim K-K, Rhim SC. Spinal osseous epidural arteriovenous fistula with multiple small arterial feeders converging to a round fistular nidus as a target of venous approach. AJNR Am J Neuroradio/ 2004;25:69-73

27. Brinjikji W, Yin R, Nasr DM, Lanzino G. Spinal epidural arteriovenous fistulas. J Neurointerv Surg 2016;8:1305-1310 